\title{
A NOTE ON SPACES IN WHICH EVERY OPEN SET IS $z$-EMBEDDED
}

\author{
JOSE L. BLASCO ${ }^{1}$
}

\begin{abstract}
Let $O z$ be the class of topological spaces in which every open set is $z$-embedded. In this note we prove the following: If $Y$ is a dense subspace of the real line, then the spaces $\beta Y$ and $\beta Y-Y$ are not in $O z$.
\end{abstract}

Introduction. A subset $S$ of a topological space $X$ is $z$-embedded in $X$ if every zero-set in $S$ is the intersection of $S$ with a zero-set in $X$. (A zero-set is the set of zeros of a real-valued continuous function.) Blair [1] studied the class $\mathrm{Oz}$ of topological spaces in which every open set is $z$-embedded. This class includes all perfectly normal spaces, all extremally disconnected spaces and all products of separable metric spaces. For basic results of the class $O z$ see [1 and 2].

Blair [1] asked if the spaces $\beta R, \beta Q$ and $\beta Q-Q$ are in $O z$. In [6] Terada characterizes a class of spaces whose Stone-Cech compactifications are in $O z$. As an application of his characterizations he showed that both $\beta R$ and $\beta Q$ do not belong to $O z$. E. K. van Douwen [4] has proved that $\beta Q-Q$ does not belong to $O z$.

In this note we shall prove that for $Y$ dense in $R$, the spaces $\beta Y$ and $\beta Y-Y$ are not in $O z$.

Preliminaries. Throughout this paper we adopt the notation and terminology of [5]. $\beta X$ and $v X$ denote respectively the Stone-Cech compactification and the Hewitt realcompactification of the Tychonoff space $X . Z(X)$ denotes the family of all zero-sets in $X$. The remainder $\beta X-X$ is always denoted by $X^{*} . R$ is the space of all real numbers with the usual topology, $Z$ is the subspace of all integer numbers and $N$ is the subspace of all positive integers.

Let $S$ be a subset of the topological space $X$. The $G_{\delta}$-closure of $S$ is the set $G_{\delta}$-cl $X$ of all points $p \in X$ satisfying the condition that whenever $G$ is a $G_{\delta}$-set containing $p$, then $G \cap S \neq \emptyset$. For Tychonoff $X, G_{\delta}-\operatorname{cl}_{X} S$ is precisely all $p \in X$ for which each zero-set about $p$ meets $S$. The following fact is needed: (a) [3,1.1(b)] If $S$ is $z$-embedded in the Tychonoff space $X$, then the $G_{\delta}$-closure of $S$ in $v X$ is $v S$. The set $S$ is said to be $G_{\delta}$-dense in $X$ if $X=G_{\delta}-\operatorname{cl}_{X} S$.

The result. In the sequel, $Y$ will be a dense subspace of $R$. Let $S=\{a(n): n \in$ $Z$ \} be a copy of $Z$ contained in $Y$ such that $a(n+1)-a(n) \geq 1$ for $n \in Z$. Consider the following closed subsets of $Y, I=\bigcup\{[a(2 n), a(2 n+1)] \cap Y: n \in Z\}$ and $J=\bigcup\{[a(2 n-1), a(2 n)] \cap Y: n \in Z\}$. Since $Y$ is a metric space, $I$ and $J$ are zero-sets in $Y$. Therefore $\beta Y=\operatorname{cl}_{\beta Y} I \cup \operatorname{cl}_{\beta Y} J$ and $\operatorname{cl}_{\beta Y} S=\operatorname{cl}_{\beta Y} I \cap \operatorname{cl}_{\beta Y} J$. We

Received by the editors January 12, 1981 and, in revised form, August 9, 1981.

1980 Mathematics Subject Classification. Primary 54D40, 54G20.

Key words and phrases. $G_{\delta}$-closure, $\mathrm{Oz}, z$-embedding.

${ }^{1}$ The author wishes to thank the referee for his suggestions.

(C) 1982 American Mathematical Society 0002-9939/81/0000-0754/\$01.75 
need the following fact:

(b) $\quad \operatorname{cl}_{\beta Y} S-S \subset \operatorname{cl}_{Y^{*}}\left(\left(\operatorname{cl}_{\beta Y} I-\operatorname{cl}_{\beta Y} S\right) \cap Y^{*}\right) \cap \operatorname{cl}_{Y^{*}}\left(\left(\operatorname{cl}_{\beta Y} J-\operatorname{cl}_{\beta Y} S\right) \cap Y^{*}\right)$.

Indeed, let $p$ be a point in $\operatorname{cl}_{\beta Y} S-S$ and let $V$ be a closed neighborhood of $p$ in $Y^{*}$. There exists an open set $W$ in $\beta Y$ such that $p \in W$ and $Y^{*} \cap \operatorname{cl}_{\beta Y} W \subset V$. Since the set $W \cap S$ is infinite, we can choose a closed (in $Y$ ) copy $E$ of $N$ such that $E \subset I \cap W$ and $E \cap S=\emptyset$. Then

$$
\emptyset \neq Y^{*} \cap \operatorname{cl}_{\beta Y} E \subset Y^{*} \cap \operatorname{cl}_{\beta Y} I \cap \operatorname{cl}_{\beta Y} W \subset\left(\operatorname{cl}_{\beta Y} I\right) \cap V .
$$

Since $E$ and $S$ are disjoint zero-sets in $Y$ it follows that $\operatorname{cl}_{\beta Y} E \cap \operatorname{cl}_{\beta Y} S=\emptyset$ and therefore the set $\left(\operatorname{cl}_{\beta Y} I-\operatorname{cl}_{\beta Y} S\right) \cap V$ is nonempty. Hence

$$
p \in \operatorname{cl}_{Y^{*}}\left(\left(\operatorname{cl}_{\beta Y} I-\operatorname{cl}_{\beta Y} S\right) \cap Y^{*}\right) .
$$

We can replace $I$ by $J$ in the above argument. The inclusion is now proved.

Let $X=\beta Y-\operatorname{cl}_{\beta Y} S$.

ASSERTION 1. $X$ is not $C^{*}$-embedded in $\beta Y-S$.

Proof. The family $\left\{\operatorname{cl}_{\beta Y} I-\operatorname{cl}_{\beta Y} S, \operatorname{cl}_{\beta Y} J-\operatorname{cl}_{\beta Y} S\right\}$ is a partition of $X$, so the characteristic function (in $X$ ) $f$ of the set $\operatorname{cl}_{\beta Y} I-\operatorname{cl}_{\beta Y} S$ is continuous on $X$. According to (b), $f$ has no continuous extension to $\beta Y-S$, therefore $X$ is not $C^{*}$-embedded in $\beta Y-S$.

Assertion 2. The $G_{\delta}$-closure in $\beta Y$ of $Y^{*} \cap X$ is $Y^{*}$.

Proof. Since the points of $Y$ are zero-sets in $\beta Y$, it follows that

$$
G_{\delta}-\operatorname{cl}_{\beta Y}\left(Y^{*} \cap X\right) \subset Y^{*} \text {. }
$$

Suppose now that $p \in Y^{*}$ is not in $G_{\delta}-\operatorname{cl}_{\beta Y}\left(Y^{*} \cap X\right)$. Then there exists a zero-set $T$ in $\beta Y$ such that $p \in T \subset \beta Y-\left(Y^{*} \cap X\right)$. Moreover, since $Y$ is realcompact [5, Corollary 8.15] there is a zero-set $F$ in $\beta Y$ such that $p \in F \subset Y^{*}$. Let $h$ be a real-valued continuous function on $\beta Y$ such that $h^{-1}(\{0\})=T \cap F \subset Y^{*} \cap \operatorname{cl}_{\beta Y} S$. The reciprocal $g$ of $h \mid X \cup S$ is continuous and unbounded on $X \cup S$, consequently $g$ must be unbounded on some countable closed subspace $H$ of $Y$ which misses $S$. Since $H$ and $S$ are disjoint zero-sets in $Y$ we have that $\operatorname{cl}_{\beta Y} H \cap \operatorname{cl}_{\beta Y} S=\emptyset$, therefore $g$ must be unbounded on $\operatorname{cl}_{\beta Y} H \subset X \cup S$, which is a contradiction. This shows $Y^{*}=G_{\delta}-\operatorname{cl}_{\beta Y}\left(Y^{*} \cap X\right)$.

Assertion 3. The space $\beta Y$ does not belong to $O z$.

Proof. Suppose that $\beta Y \in O z$. Then $X$ is $z$-embedded in $\beta Y$ and according to (a), $v X=G_{\delta}-\operatorname{cl}_{\beta Y} X$. Since the points of $Y$ are zero-sets in $\beta Y$, it follows that $v X \subset \beta Y-S$. From Assertion 2 we have $v X=\beta Y-S$, which contradicts Assertion 1. Hence $\beta Y \notin O z$.

A subset $S$ of a space $E$ is a generalized cozero-set in case for every neighborhood $V$ of $S$ there is a cozero-set $P$ such that $S \subset P \subset V$. It is known that every generalized cozero-set in a normal space is normal and $z$-embedded $[1$, Theorem $2.5]$.

ASSERTION 4. $Y^{*}$ is realcompact and $z$-embedded in $\beta Y$. 
Proof. Since every compact subset of $Y$ is a zero-set in $\beta Y$, we have that $Y^{*}$ is a generalized cozero-set in $\beta Y$. According to [1, Theorem 2.5], $Y^{*}$ is $z$-embedded in $\beta Y$. On the other hand, since every cozero-set in $\beta Y$ is realcompact and $Y^{*}=$ $\bigcap\{\beta Y-\{p\}: p \in Y\}$, it follows that $Y^{*}$ is realcompact.

ASSERTION 5. $Y^{*}$ does not belong to $O z$.

Proof. From Assertion $4, Y^{*}$ is $z$-embedded in $\beta Y$. Thus by Assertion 2, $G_{\delta}-\operatorname{cl}_{Y^{*}}\left(Y^{*} \cap X\right)=Y^{*}$. Hence, if $Y^{*} \in O z$ we have that $Y^{*} \cap X$ is $G_{\delta}$-dense and $z$-embedded in $Y^{*}\left[1\right.$, Theorem 5.1]. Therefore $Y^{*}=v\left(Y^{*} \cap X\right)$ and $Y^{*} \cap X$ is $C$-embedded in $Y^{*}$.

On the other hand, the set $\left(\operatorname{cl}_{\beta Y} I-\operatorname{cl}_{\beta Y} S\right) \cap Y^{*}$ is clopen in $Y^{*} \cap X$, therefore its characteristic function (in $Y^{*} \cap X$ ) is continuous. According to (b), this function has no continuous extension to $Y^{*}$. This contradiction shows that $Y^{*} \notin O z$.

\section{REFERENCES}

1. R. L. Blair, Spaces in which special sets are z-embedded, Canad. J. Math. 28 (1976), 673-690.

2. R. L. Blair, Cech-Stone remainders of locally compact nonpseudocompact spaces, Topology Proc. 4 (1979), 13-17.

3. R. L. Blair and A. W. Hager, Notes on the Hewitt realcompactification of a product, Gen. Topology Appl. 5 (1975), 1-8.

4. E. K. van Douwen, The Cech-Stone remainder of some nowhere locally compact spaces, manuscript.

5. L. Gillman and M. Jerison, Rings of continuous functions, Van Nostrand, Princeton, N.J., 1960.

6. T. Terada, On spaces whose Stone-Cech compactification is Oz, Pacific J. Math. 85 (1979), 231-237.

Catedra de matematicas II, Facultad de Ciencias, Burjasot, Valencia, SPAIN 